\section{Experiencias de estudiantes de medicina que interrumpen sus estudios universitarios: un estudio cualitativo}

\author{
MARY JANE SCHILLING-NORMAN ${ }^{1, a}$, JAVIERA ORTEGA-BASTIDAS \\ CRISTHIAN PÉREZ-VILLALOBOS ${ }^{1, \mathrm{c}}$, ALEJANDRO DÍAZ-MUJICA $^{2, \mathrm{~d}}$
}

\section{Experiences of medical students dropping out studies. A qualitative approach}

Background: Medical students have a higher prevalence of mental health problems and are more likely to drop out their studies as a result. Aim: To comprehend the academic experiences of medical students who dropped out medical studies in a university that experienced an increase in drop out by medical students and consultations to mental health services. Material and Methods: A qualitative study with a phenomenological design, in which in-depth interviews were conducted with eight medical students who dropped out studies. The analysis was carried out by means of the constant comparison method up to the level of open coding. Results: Two categories were identified, namely experiences prior to interrupting studies, which comprised the subcategories of academic and social experiences, and motives that lead to drop-out, which considered the subcategories related to vocation, mental health and pursuing other projects. Conclusions: These results identified the challenges faced by students prior to stopping-out, that are related to academic aspects, peer relations and student-teacher relationship. The main motives for drop-out reported by students were mental health problems.

(Rev Med Chile 2021; 149: 1215-1222)

Key words: Mental Health; Student Dropouts; Students, Medical.
Departamento de Educación Médica, Facultad de Medicina, Universidad de Concepción. Concepción, Chile.

2Departamento de Psicología, Facultad de Ciencias Sociales, Universidad de Concepción.

Concepción, Chile.

apsicóloga, Magíster en

Psicología, mención Psicología

Educativa.

bPsicóloga, Doctora ${ }^{\circledR}$ en Ciencias

Sociales.

'Psicólogo, Doctor en Ciencias de la Educación.

¿Psicólogo, Doctor en Psicología.

Este trabajo fue financiado por Agencia Nacional

de Investigación y

Desarrollo/ Programa

Becas/ Beca de Magíster Nacional/2019-22190605. Los autores declaran no tener conflictos de interés.

Recibido el 3 de diciembre de 2020, aceptado el 27 de septiembre de 2021.

Correspondencia a: Mary Jane Schilling-Norman Departamento de Educación Médica, Facultad de Medicina, Universidad de Concepción. Concepción, Chile. maryschilling@udec.cl
$\mathrm{D}$ esde el año 2009, las universidades chilenas han orientado sus esfuerzos a favorecer la permanencia de los estudiantes en los programas de estudio ${ }^{4}$.

La interrupción de estudios contempla aquellos estudiantes que han suspendido sus estudios un semestre o más y que luego retoman su formación académica ${ }^{5}$.

Durante el primer año de la carrera de Medicina, en Chile, cerca de $6 \%$ de los estudiantes abandonan sus estudios y $4 \%$ se reincorpora al año siguiente ${ }^{6}$. Sin embargo, en estos análisis, no se ha tomado en consideración datos estadísticos sobre años posteriores.

Se ha evidenciado que algunos de los factores que son determinantes para la interrupción de estudios universitarios son las dificultades en el rendimiento académico, dificultades en la adaptación social, tener que trabajar mientras se estudia, y presentar problemas financieros ${ }^{7}$.

Particularmente, en el caso de Medicina, la evidencia a nivel mundial muestra que a medida que el estudiante progresa en la carrera, la posibilidad de retirarse por problemas de salud mental aumenta y constituye un factor significativo entre las razones de abandono ${ }^{3,8-11}$, reportando baja calidad de vida, depresión y burnout académico ${ }^{12}$.

Considerando la amplia evidencia respecto a la alta prevalencia de enfermedades de salud mental en los estudiantes de Medicina a nivel mundial, 
relacionado con depresión, ansiedad y burnout $t^{1,2,13}$; y el papel que juegan las experiencias universitarias sobre la salud mental de sus estudiantes ${ }^{14-17}$, sumado al aumento de consultas en salud mental e interrupción de estudios en Medicina según información entregada por secretaría académica y Unidad de Apoyo al Estudiante de la casa de estudios donde se realizó la investigación, surgió la interrogante ¿Cuáles son las experiencias universitarias de los estudiantes de Medicina que interrumpen su formación académica?

El objetivo general de este estudio fue comprender las experiencias universitarias de los estudiantes de Medicina que interrumpen su formación académica. Los objetivos específicos fueron: describir las experiencias a nivel académico y social previo a interrumpir estudios y describir los motivos que llevan a los estudiantes a tomar la decisión de interrumpir estudios. Este artículo considera una parte de los resultados obtenidos en la investigación que se centró en indagar todo el proceso que vive el estudiante durante su interrupción de estudios y posterior reincorporación.

\section{Material y Métodos}

Se realizó un estudio cualitativo con diseño fenomenológico ${ }^{18}$. La fenomenología busca conocer los significados que los individuos dan a su experiencia, para así aprehender el mundo tal como lo construye la otra persona ${ }^{19}$.

La muestra estuvo compuesta por estudian- tes de la carrera de Medicina de una universidad chilena. Dentro de los criterios de inclusión se consideró que hubiesen interrumpido sus estudios universitarios previamente, o que en el momento de la recolección de datos estuviesen cursando la interrupción. Dentro de los criterios de exclusión se consideró que hubiesen interrumpido estudios junto con un cambio de carrera o de institución. Se utilizó un muestreo por conveniencia, a través de participantes voluntarios ${ }^{19}$.

De los 12 participantes que mostraron interés en el estudio, se reclutó a un total de 8 estudiantes (caracterizados en la Tabla 1), ya que otros no cumplían con criterios de inclusión o desistieron de su participación. Se cumplieron los criterios de saturación teórica al no surgir nuevas categorías dentro de las entrevistas, así como también el criterio recomendado por Creswell ${ }^{20}$ para diseños fenomenológicos, que considera entre 8 y 12 participantes.

Se utilizó la entrevista en profundidad como técnica de recolección de información, que consiste en un guión de preguntas con temas, pudiendo profundizar en otras temáticas que vayan surgiendo en la entrevista, ahondando así en los significados otorgados por los participantes ${ }^{19}$. Las entrevistas tuvieron una hora aproximada de duración y fueron realizadas por la investigadora principal por videollamada a través de $\mathrm{Zoom}^{\odot}$, considerando la contingencia del COVID-19 en nuestro país.

En cuanto al procedimiento de recolección de información, se solicitó autorización institucional al Comité Ético Científico de la Facultad de

Tabla 1. Caracterización de los participantes del estudio

\begin{tabular}{|ccccc|}
\hline Participante & Sexo & $\begin{array}{c}\text { Nivel formativo donde } \\
\text { se interrumpe estudios }\end{array}$ & $\begin{array}{c}\text { Tiempo de la } \\
\text { interrupción }\end{array}$ & $\begin{array}{c}\text { Motivo reportado de la } \\
\text { interrupción }\end{array}$ \\
\hline 1 & Femenino & Segundo año & 1 semestre & Otros proyectos \\
\hline 2 & Femenino & Tercer año & 1 año (actualmente) & Vocacional y Salud Mental \\
\hline 3 & Femenino & Sexto año & 3 meses & Salud Mental \\
\hline 4 & Femenino & Quinto año & 1 año & Salud Mental \\
\hline 5 & Masculino & Cuarto año & 1 semestre & Vocacional y Salud Mental \\
\hline 6 & Femenino & Sexto año & 3 meses & Salud Mental \\
\hline 7 & Masculino & Tercer año & 1 año & Otros proyectos \\
8 & Femenino & Tercer año & 1 año & Salud Mental \\
\hline
\end{tabular}


Medicina de la Universidad de Concepción. Una vez obtenidas las autorizaciones correspondientes se hizo un llamado voluntario a participar de la investigación por medio un afiche difundido a través de redes sociales y a través del correo institucional. Voluntariamente, cada estudiante se contactó vía correo electrónico mostrando su interés en participar en la investigación. Se le envió el consentimiento informado para revisar previo a la realización de la entrevista.

Para realizar el análisis de la información obtenida, se utilizó el método de comparación constante hasta el nivel de codificación abierta, que consiste en identificar a partir de los datos las categorías junto con sus propiedades y dimensiones, lo cual permite tener un primer acercamiento al fenómeno ${ }^{19}$.

Se aseguraron los criterios de rigor metodológico de credibilidad, transferencia, dependencia, confirmabilidad y autenticidad ${ }^{18}$, mediante transcripciones y análisis de cada entrevista inmediatamente después de su aplicación, y discusión con los participantes de los hallazgos, para evitar introducir sesgos por parte del equipo investigador.
Además, se emplearon códigos in vivo que utilizan el lenguaje propio del entrevistado.

Se empleó el software de análisis de datos cualitativos Atlas.ti versión 7.5.4.

\section{Resultados}

A partir del análisis se identificaron 2 categorías: a) Experiencias previas a la interrupción de estudios y b) Motivos que llevan a la interrupción de estudios. En la Tabla 2, se pueden revisar las subcategorías y citas para cada una de ellas.

\section{Experiencias previas a la interrupción de estudios}

Los entrevistados refirieron experiencias a nivel académico y social que debieron enfrentar a lo largo de la carrera y que configuraron el contexto en el cual se desarrolló la interrupción de estudios.

\section{Experiencias académicas}

Los participantes relataron que, previo a interrumpir sus estudios, se enfrentaban a una alta

Tabla 2. Categorías y citas

\begin{tabular}{|lll|}
\hline Categoría & Subcategoría & Cita \\
Experiencias & Experiencias & 1. P1: "Entonces, claro es como que toda la carga, incluso sin considerar lo del depor- \\
previas a la & académicas & te, solo la carga académica, a mi por lo menos me ha limitado harto como la vida \\
interrupción & social" [Entrevistado 1] \\
de estudios & 2. "(..) ya no podía ni estar despierta durante el día, así que mi médico que me trata \\
& la depre (...) me recetó modafinilo para que pudiera estar despierta en el día (...)" \\
& [Entrevistada 2] \\
3. "Es que preguntan puras cosas nada que ver, si nos iba bien solo porque nos sabía- \\
mos las preguntas, los profesores preguntabas cosas que no tenían nada que ver \\
con lo que ellos enseñaban, que eran cero relevante para saberse la materia, en \\
algunos ramos, no en todos" [Entrevistado 2] \\
4. "(...) todo el servicio recaía en nosotros, no alcanzábamos a almorzar, teníamos que \\
estudiar también, casi todos los días hacerle una presentación a un doctor...y... \\
eso, era súper estresante, súper movido, uno llegaba a la casa y había que hacer la \\
presentación... habían turnos también, por lo menos dos o tres veces a la semana \\
hasta las ocho, o sea en ese sentido, la teníamos mejor que los internados de [asig- \\
natura] y de [asignatura] pero igual era pesadísimo el servicio" [Entrevistada 3] \\
5. P3: "(...) había que entubarlo ya, entonces lo tuvieron que reingresar a pabellón \\
y todo, y eso fue recién cuando llegamos en la mañana, y no habían pasado visita \\
ni nada, y estábamos solas nosotras con mi compañera, y eso fue como un poco \\
estresante porque si uno no lo hubiera revisado al tiro el paciente se muere (...)" \\
[Entrevistada 3] \\
6. P5: "Em...por ejemplo que les dicen que no sirven para la carrera, que no tienen \\
idea de cosas básicas..." [Entrevistado 5]
\end{tabular}




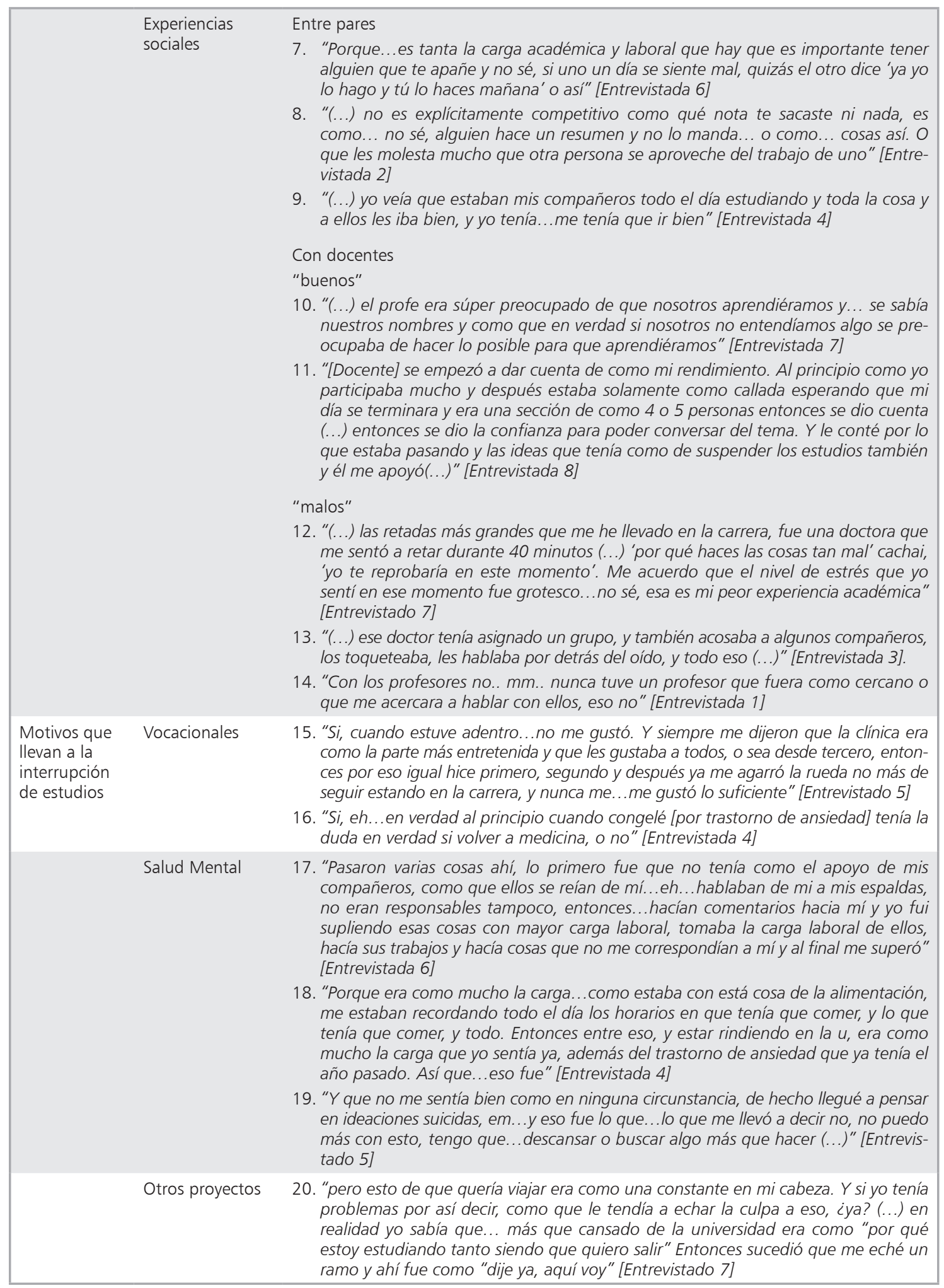


carga académica, que solía aumentar al ingresar a campos clínicos. Ellos dedicaban gran parte de su día a estudiar, afectando así su vida social, sus tiempos de ocio y sus horas de sueño (cita 1). Frente a esto, algunos entrevistados recurrían al uso de psicofármacos, con la finalidad de rendir adecuadamente (cita 2). Pese a esto, la mayor parte de los estudiantes entrevistados mantenían un buen rendimiento académico.

En cuanto a las evaluaciones, estas eran consideradas una fuente de estrés para los participantes, ya que percibían que el docente preguntaba aspectos que iban más allá de los conocimientos esperados en una asignatura (cita 3).

Específicamente en el internado, los entrevistados se sentían estresados, ya que la carga de trabajo era pesada, al incluir turnos, tener que levantarse temprano y, además, dedicar tiempo para estudiar posterior a las clases y rotaciones (cita 4). También mencionaron que gran parte del servicio recaía sobre ellos, con poca supervisión de las actividades que realizaban y una deficiente enseñanza por parte de los tutores clínicos (cita 5).

El estrés percibido era aun mayor en el caso de las evaluaciones, ya que los estudiantes referían que, tanto en los exámenes orales como en las interrogaciones durante las rotaciones, debían enfrentarse al miedo de vivir experiencias de lo que ellos denominaban humillaciones por parte de los docentes, cuando no sabían o respondían mal a alguna pregunta, donde los docentes los ridiculizaban y les cuestionaban su pertenencia a la carrera (cita 6).

\section{Experiencias sociales}

Los estudiantes refirieron experiencias sociales previas a interrumpir sus estudios, tanto con sus compañeros, como con sus docentes.

\section{Experiencias sociales con compañeros}

Los estudiantes refirieron que previo a interrumpir estudios tenían buenas relaciones con sus compañeros de curso. Además, identificaban prácticas de apoyo entre compañeros, tales como prestarse material de estudio, apadrinar compañeros de cursos inferiores, y brindar apoyo emocional, lo cual hacía el estrés universitario más llevadero (cita 7).

Paralelo a lo anterior, también se identificaba competencia entre compañeros por tener un buen rendimiento académico, lo cual generaba una presión por rendir bien (cita 8 y 9). A su vez, los participantes relataron situaciones de discriminación entre compañeros, así como también situaciones de abuso sexual, lo cual hacía peligrar las relaciones dentro del curso y generaba hostilidad entre algunos compañeros (cita 17).

\section{Experiencias sociales con docentes}

Los estudiantes entrevistados evaluaron la relación docente-estudiante previo a interrumpir estudios en el ámbito académico, diferenciando docentes "buenos" y "malos". Aquellos "docentes buenos" empleaban estrategias de enseñanza-aprendizaje que facilitaban el aprendizaje de los estudiantes y ellos valoraban que el docente supiera el nombre de los estudiantes y respondiera a sus dudas (cita 10).

En cuanto a los "docentes malos", los estudiantes percibían que no aprendían en sus clases, que las evaluaciones no eran justas al evaluar aspectos que estaban fuera del programa de asignatura, o no retroalimentaban su desempeño. Los estudiantes reportaron también que algunos docentes presentaban conductas de maltrato, acoso sexual y abuso de poder. Se mencionaron en las entrevistas situaciones en donde los estudiantes eran menospreciados y humillados por no saber toda la materia (cita 12), y situaciones en donde los docentes tenían un trato inadecuado con los estudiantes, sin respetar su privacidad y espacio personal (cita 13). Pocas veces se denunciaron dichas acciones por temor a represalias, y porque se percibía que las denuncias no generaban consecuencias para los docentes.

En general, los docentes no eran percibidos como personas cercanas (cita 14), se mencionó la existencia de una jerarquía que dificulta el encuentro, es decir, el poder acercarse al docente y poder hablar más allá de lo académico. A pesar de esto, los estudiantes destacaron a algunos docentes y autoridades de la facultad como personas más cercanas, preocupadas por el desarrollo emocional de sus estudiantes y quienes fueron un apoyo al tomar la decisión de interrumpir sus estudios (cita 11).

\section{Motivos que llevan a la interrupción de estudios}

Los estudiantes entrevistados mencionaron tres principales motivos que les llevaron a interrumpir sus estudios: dudas vocacionales, salud mental y el deseo de desarrollar otros proyectos. 
En cuanto a las dudas vocacionales, los estudiantes entrevistados que interrumpieron por este motivo planteaban que desde antes de entrar a la carrera tenían dudas respecto a si les gustaba Medicina. En el proceso de la carrera, los estudiantes relataron dificultades para poder estudiar y rendir académicamente al tener esta duda vocacional, sintiéndose frustrados, estresados y descontentos (cita 15). Otros estudiantes mencionaron que, si bien las dudas vocacionales no eran su principal motivo para interrumpir estudios, sí las tuvieron en algún momento de la carrera (cita 16).

Los estudiantes también relataron diferentes problemas de salud mental que llevaron a interrumpir sus estudios universitarios. Estos tenían que ver principalmente con diagnósticos de depresión, ansiedad y burnout. Los participantes entrevistados comentaron que el estrés, las dificultades con compañeros y la carga de la universidad hizo que desarrollaran estas patologías o que se exacerbara la sintomatología que estaban desarrollando, por lo que decidieron interrumpir sus estudios para poder centrarse en recuperar su salud mental (citas 17 y 18). Otros estudiantes que tenían dudas vocacionales también desarrollaron enfermedades de salud mental, lo cual fue un agravante que influyó en la toma de esta decisión (cita 19).

Otros estudiantes decidieron interrumpir sus estudios universitarios para poder llevar a cabo otros proyectos como, por ejemplo, viajar o dedicarse al deporte profesionalmente. Estas actividades eran incompatibles con los estudios universitarios, lo cual motivó la decisión en ese momento, para poder dedicar tiempo a ellas (cita 20).

\section{Discusión}

La presente investigación buscó dar respuesta a la pregunta de investigación ¿Cuáles son las experiencias universitarias de los estudiantes de Medicina que interrumpen su formación académi$c a$ ?, identificando las categorías de experiencias previas a interrumpir estudios y motivos de la interrupción de estudios.

A partir de los resultados obtenidos en esta y otras investigaciones, las experiencias de los estudiantes que interrumpen sus estudios universitarios convergen en un contexto común relacionado con el ámbito pedagógico y la relación docente-estudiante. Estos elementos ponen una carga considerable de estrés sobre los estudiantes, influyendo sobre su decisión de interrumpir estudios ${ }^{1,9,16,21,22}$.

Tanto este estudio como otras investigaciones concuerdan que los estresores asociados a la carrera de Medicina están relacionados con la alta carga académica, las evaluaciones orales y deficiencias en el proceso de enseñanza-aprendizaje ${ }^{1,8,16,21,22}$.

Además, existe un consenso a nivel mundial respecto a la presencia de diferentes tipos de maltrato en las escuelas de Medicina, en donde se han reportado humillaciones, menoscabo, acoso sexual y discriminación. Se destaca también en esta y otras investigaciones el miedo a la denuncia por parte del estudiante y la baja percepción de las consecuencias que pudiese generar sobre el docente $e^{23-27}$.

Las relaciones sociales entre compañeros son consideradas por los entrevistados como un soporte emocional y, a la vez, un estresor en la carrera. Evidencia empírica muestra que las relaciones sociales son un factor protector dentro de la vida universitaria ${ }^{17,28-31}$, ya que los estudiantes comparten en común las dificultades de la carrera y se brindan apoyo y soporte mutuamente, tanto a nivel académico como emocional ${ }^{32}$.

Pese a lo anterior, igualmente hay situaciones entre pares que se presentan como nocivas para el establecimiento de las relaciones y el manejo del estrés, como lo es el ambiente de competencia que se genera entre los compañeros, así como también situaciones de abuso y maltrato entre pares, realidades que también son observadas en otras escuelas de Medicina ${ }^{2,14,15}$.

Los estudiantes entrevistados consideran estos antecedentes institucionales en muchas ocasiones como causantes o agravantes de su salud mental. De los ocho estudiantes entrevistados, seis interrumpieron estudios por motivos de salud mental. Esto es concordante con otras investigaciones que dan cuenta que este factor es uno de los principales motivos para abandonar o interrumpir la carrera de Medicina ${ }^{3,7-9}$.

En este estudio se pudo observar que la salud mental es un eje central en los motivos para interrumpir estudios y no se presenta como un fenómeno aislado. Las dudas vocacionales se ven teñidas por problemas de salud mental, y la preocupación por el bienestar aparece en el deseo de desarrollar otros proyectos personales. 
Igualmente, las dudas vocacionales aparecieron en algunos estudiantes que interrumpieron por motivos de salud mental.

Los factores institucionales, tales como las experiencias de enseñanza-aprendizaje y el ambiente educativo, afectan la salud mental de los estudiantes, pudiendo agravar condiciones preexistentes o ser un factor contextual que facilita el desarrollo de problemas de salud mental.

Los resultados de la presente investigación pueden ser una contribución a la comprensión de la interrupción de estudios en Medicina e invitan a profundizar y discutir entre los diferentes actores de las instituciones de educación superior, la temática de la salud mental y su influencia sobre la permanencia de estudios.

Respecto a las limitaciones de este estudio, la muestra, si bien permitió saturar teóricamente las categorías, su representatividad se limita a una universidad. Futuras investigaciones deberían considerar incluir una muestra más variadas en términos de sus universidades o su zona geográfica.

En cuanto a futuras líneas de investigación, dado que la interrupción de estudios es una temática poco abordada tanto en Chile como en el mundo, en contraposición a la deserción, futuras investigaciones podrían contribuir ahondando sobre qué sucede con la interrupción de estudios en otras carreras de la salud y considerar estudios multicéntricos, que comparen diferentes experiencias de acuerdo a las diferentes universidades y carreras, así como también incluir muestras que consideren tanto a estudiantes como docentes.

\section{Referencias}

1. Hope V, Henderson M. Medical student depression, anxiety and distress outside North America: a systematic review. Med Educ. 2014; 48 (10): 963-79.

2. Rotenstein L, Ramos M, Torre M, Segal B, Peluso M, Guille C, et al. Prevalence of depression, depressive symptoms, and suicidal ideation among medical students a systematic review and meta-analysis. J Am Med Assoc. 2016; 316 (21): 2214-36.

3. Adhikari A, Dutta A, Sapkota S, Chapagain A, Aryal A, Pradhan A. Prevalence of poor mental health among medical students in Nepal: A cross-sectional study. BMC Med Educ. 2017; 17 (1): 1-7.

4. Faúndez R, Labarca JP, Cornejo MF, Villarroel M, Gil F. Ranking 850, transición a la educación terciaria de estudiantes con desempeño educativo superior y puntaje PSU insuficiente. Pensam Educ Rev Investig Educ Latinoam. 2017; 54 (1): 1-11.

5. Bonham A, Luckie J. Community college retention: Differentiating among stopouts, dropouts, and optouts. Community Coll J Res Pract. 1993; 17 (6): 543-54.

6. SIES. Deserción de primer año y Reingreso a la Educación Superior en Chile. Santiago, Chile 2019.

7. Woosley S. Stop-Out or Drop-Out? An Examination of College Withdrawals and Re-Enrollments. J Coll Student Retent. 2003; 5 (3): 293-303.

8. Arulampalam W, Naylor R, Smith J. Factors affecting the probability of first year medical student dropout in the UK: A logistic analysis for the intake cohorts of 1980-92. Med Educ. 2004; 38 (5): 492-503.

9. Dyrbye L, Thomas M, Shanafelt T. Systematic review of depression, anxiety, and other indicators of psychological distress among U.S. and Canadian medical students. Acad Med. 2006; 81 (4): 354-73.

10. Maher B, Hynes H, Sweeney C, Khashan A, O’Rourke $\mathrm{M}$, Doran K, et al. Medical school attrition-beyond the statistics a ten year retrospective study. BMC Med Educ. 2013; 13.

11. Yates J. When did they leave, and why? A retrospective case study of attrition on the Nottingham undergraduate medical course. BMC Med Educ. 2012; 12 (1).

12. Dyrbye LN, Thomas MR, Power DV, Durning S, Moutier C, Massie FS, et al. Burnout and serious thoughts of dropping out of medical school: A multi-institutional study. Acad Med. 2010; 85 (1): 94-102.

13. Miranda-Ackerman R, Barbosa-Camacho F, Sander-Möller M, Buenrostro-Jiménez A, Mares-País R, Cortes-Flores A, et al. Burnout syndrome prevalence during internship in public and private hospitals: a survey study in Mexico. Med Educ. Online [Internet] 2019; 24 (1). Available from: https://doi.org/10.1080/10 872981.2019.1593785.

14. Hu K, Chibnall J, Slavin S. Maladaptive Perfectionism, Impostorism, and Cognitive Distortions: Threats to the Mental Health of Pre-clinical Medical Students. Acad Psychiatry. 2019; 43: 381-5.

15. Slavin S, Schindler D, Chibnall J. Medical student mental health 3.0: Improving student wellness through curricular changes. Acad Med. 2014; 89 (4): 573-7.

16. Gutiérrez J, Montoya L, Toro B, Briñón MA, Rosas E, Salazar L. Depresión en estudiantes universitarios y su asociación con el estrés académico. Rev CES Med [Internet]. 2010; 24 (1): 7-17. Available from: http://www. redalyc.org/articulo.oa?id=261119491001\%0ACómo

17. Pérez C, Maldonado P, CA, Acosta M. Clima Educativo y su Relación con la Salud Mental de Alumnos Universi- 
tarios Chilenos. Rev Argentina Clínica Psicológica. 2013; 22 (3): 257-68.

18. Hays D, Singh A. Qualitative Inquiry in Clinical and Educational Settings. New York: The Guildford Press 2012.

19. Rodríguez G, Gil J, García E. Metodología de la Investigación Cualitativa. 2a Edición. Málaga: Ediciones Aljibe 1999.

20. Creswell J. Qualitative Inquiry \& Research Design: Choosing Among Five Approaches. $3^{\circ}$ Edición. California: Sage 2006.

21. Mahroon ZA, Borgan SM, Kamel C, Maddison W, Royston M, Donnellan C. Factors Associated with Depression and Anxiety Symptoms Among Medical Students in Bahrain. Acad Psychiatry. 2018; 42 (1): 31-40.

22. Tomaz S, Silva R, Heath N. Estresse na Formação médica: como Lidar com Essa realidade? Rev Bras Educ Med. 2015; 39 (4): 558-64.

23. Broad J, Matheson M, Verrall F, Taylor A, Zahra D, Alldridge L, et al. Discrimination, harassment and non-reporting in UK medical education. Med Educ. 2018; 52 (4): 414-26.

24. Chung M, Thang C, Vermillion M, Fried J, Uijtdehaage S. Exploring medical students' barriers to reporting mistreatment during clerkships: a qualitative study. Med Educ Online [Internet]. 2018; 23 (1). Available from: https://doi.org/10.1080/10872981.2018.1478170.

25. Hasty B, Miller S, Bereknyei S, Lin D, Shipper E, Lau J. Medical student perceptions of a mistreatment program during the surgery clerkship. Am J Surg. [Internet] 2018; 215 (4): 761-6. Available from: https://doi.org/10.1016/j. amjsurg.2018.01.001.

26. Sánchez N, Bonilla L, Rodríguez M, Sandoval G, Alzate J, Murcia N, et al. Frequency of bullying perceived in clinical practices of last year interns of a medicine school: Cross sectional study. Rev Fac Med. 2016; 64 (3): 447-52.

27. Paredes OL, Sanabria Ferrand PA, González Quevedo LA, Moreno Rehalpe SP. "Bullying” en las facultades de medicina colombianas, mito o realidad. Rev Med. 2010; 18 (2): 161.

28. De Luca SM, Franklin C, Yueqi Y, Johnson S, Brownson C. The Relationship Between Suicide Ideation, Behavioral Health, and College Academic Performance. Community Ment Health J. 2016; 52 (5): 534-40.

29. Megivern D, Pellerito S, Mowbray C. Barriers to Higher Education for Individuals with Psychiatric Disabilities. Psychiatr Rehabil J. 2003; 26 (3): 217-31.

30. Dyrbye L, Wittlin N, Hardeman R, Yeazel M, Herrin J, Dovidio J, et al. A Prognostic Index to Identify the Risk of Developing Depression Symptoms Among U.S. Medical Students Derived From a National, Four-Year Longitudinal Study. Acad Med. 2019; 94 (2): 217-26.

31. Talih F, Daher M, Daou D, Ajaltouni J. Examining Burnout, Depression, and Attitudes Regarding Drug Use Among Lebanese Medical Students During the 4 Years of Medical School. Acad Psychiatry 2018; 42 (2): 288-96.

32. Van der Zanden P, Denessen E, Cillessen A, Meijer P. Domains and predictors of first-year student success: A systematic review. Educ Res Rev. [Internet] 2018; 23: 57-77. Available from: https://doi.org/10.1016/j. edurev.2018.01.001. 